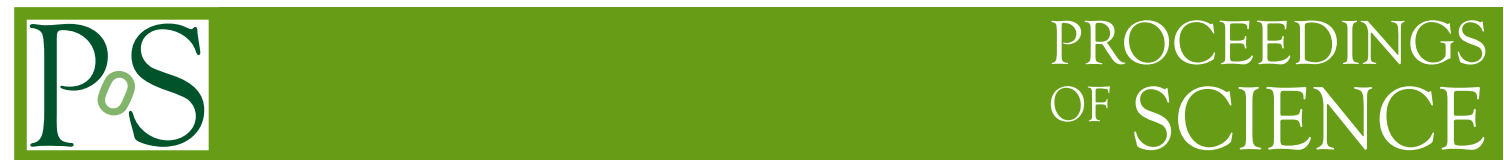

\title{
Analysing very large datasets with ParselTongue
}

\author{
S. Bourke, ${ }^{a}$ H. J. van Langevelde, ${ }^{b}$ L. Harvey-Smith, ${ }^{b}$ and A. Golden ${ }^{a}$ \\ ${ }^{a}$ National University of Ireland, Galway \\ ${ }^{b}$ Joint Institute for VLBI in Europe \\ E-mail: stephen@it.nuigalway.ie
}

\begin{abstract}
With the ever increasing bandwidth of radio receivers and correlators, the task of analysing the resulting datasets grows accordingly. The EVN was recently used to observe several methanol maser sites at high time and frequency resolution yielding $>2$ arc-minute fields of view at milliarcsecond resolution.

We present here work on the utilisation of the ParselTongue environment to automate the searching of these fields for maser sources and include initial results from work pertaining to the starforming regions $\mathrm{W} 3(\mathrm{OH})$ and Cep $\mathrm{A}$.
\end{abstract}

8th European VLBI Network Symposium

September 26-29, 2006

Toruń, Poland 

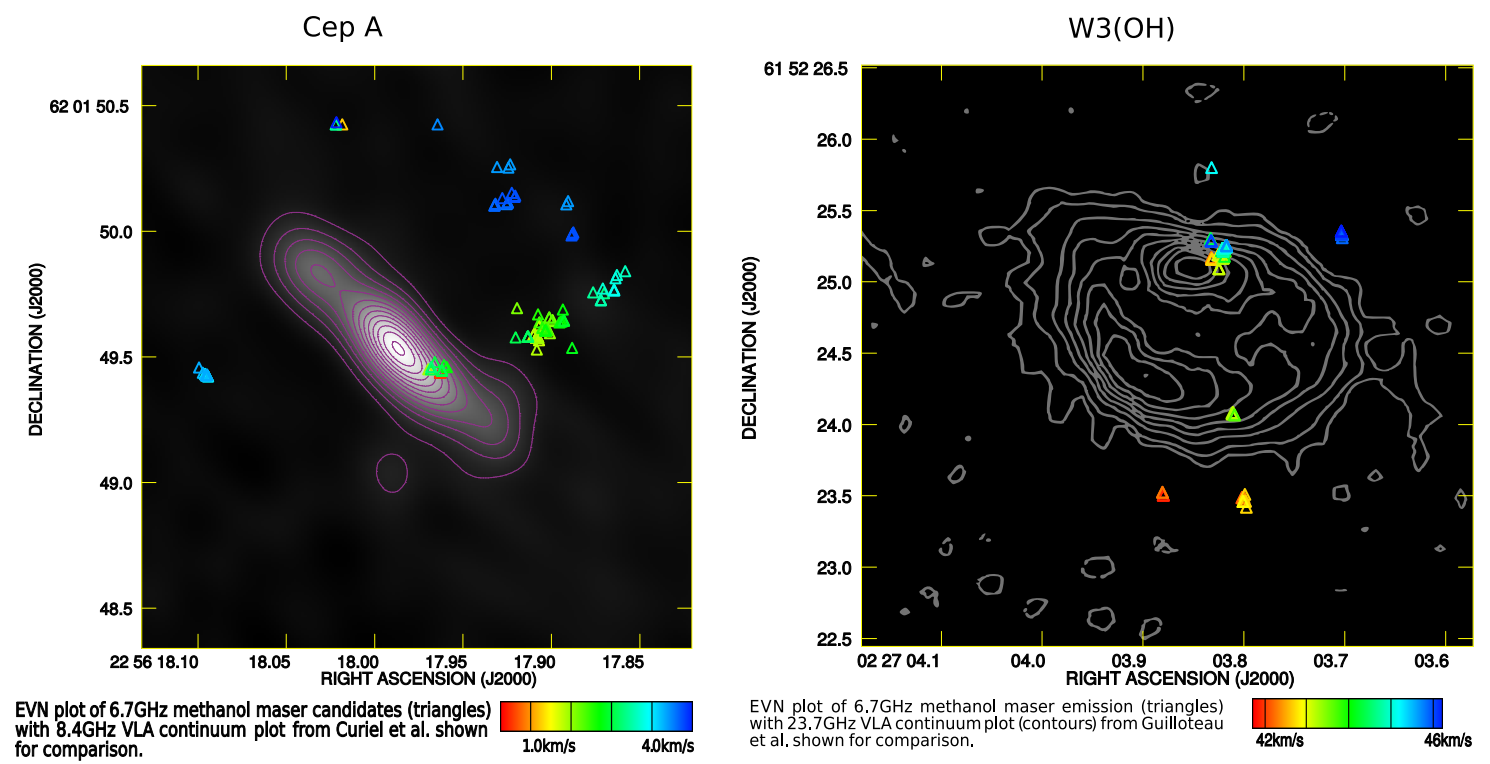

Figure 1: Plots produced from automated processing.

\section{Dataset}

In November 2004, thirteen methanol maser sites were observed with the EVN over a period of one day, using the stations: Medicina, Onsala, Torun, Cambridge, Darnhall, Noto, Effelsberg, and Westerbork. The observation contained 1024 channels centred at $6.669 \mathrm{GHz}$ and used 0.25 second integrations. The resulting dataset was $\sim 300 \mathrm{~GB}$. Due to the high frequency and time resolution, very wide fields of view are obtainable with these observations. For this reason an automated method of detecting sources in the outer field was desirable.

\section{Implementation}

The task of searching for maser sources in the data was implemented in Python using the ALBUS $^{1}$ project's ParselTongue ${ }^{2}$ interface to AIPS. The dataset was initially calibrated with ParselTongue, it was then inspected manually, and some editing, and re-calibration was done. Once the data is in an imageable state ParselTongue is utilised for the detection work.

\section{Future Work}

To date, the inner 10" of two fields have been searched. This represents a small fraction of the data. Searching the entire dataset is a computationally non-trivial task. Initial work in being carried out on a parallel implementation of the detection system. ParselTongue's in-built remote disk/execution abilities facilitate this goal. A more extensive analysis of maser structure is also in development with the aim of more accurately identifying sources.

\footnotetext{
${ }^{1}$ Advanced long baseline user software

${ }^{2}$ http://www.radionet-eu.org/rnwiki/ParselTongue
} 


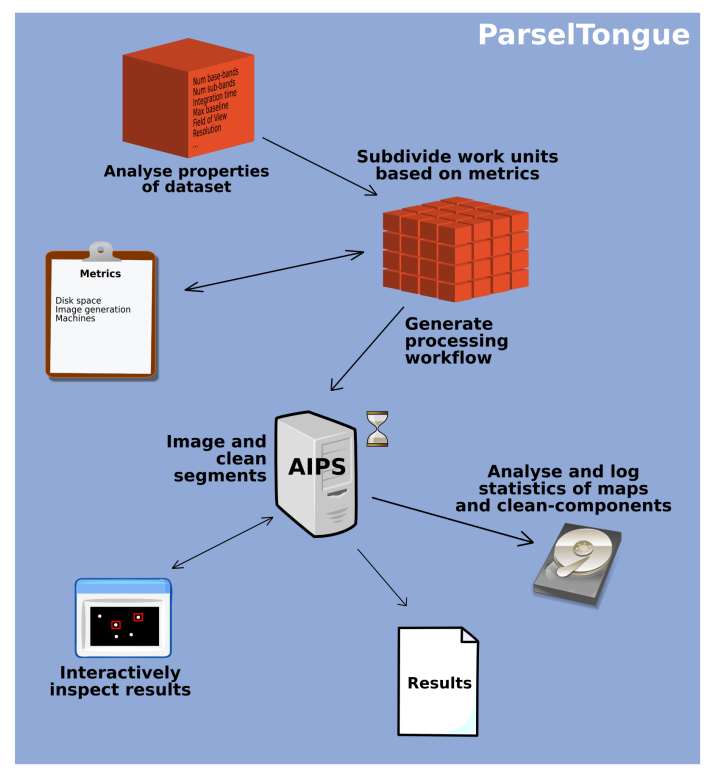

Figure 2: Algorithm and Workflow. 